\title{
INCLUSION BODIES IN ORAL DISEASES - AN OVERVIEW
}

\section{Dr. M.Parasakthi}

PG. Student, Department of Oral Pathology and Microbiology, Sree Balaji Dental College and Hospital

Article Info: Received 04 October 2020; Accepted 14 November. 2020

DOI: https://doi.org/10.32553/jbpr.v9i5.809

Corresponding author: Dr. M. Parasakthi

Conflict of interest statement: No conflict of interest

\section{ABSTRACT:}

The inclusion bodies are tiny particles found freely suspended and floating within the cytoplasmic matrix. Due to this, it is also called as cytoplasmic inclusions. These are formed with decreasing $\mathrm{pH}$ and from the pool of soluble fusion proteins within the cell. They are the elementary bodies, formed during infectious diseases or within the virus-infected cells such as rabies, herpes, measles, etc. Disease progression occurs with biochemical and cellular changes. Inclusion bodies are used in staging the diseases and aid in planning the treatment. This article aims to review the inclusion bodies in various oral lesions and their morphology.

Keywords: Inclusion body, Physiological, Pathological, Oral lesions.

\section{Introduction}

\section{Classification of Inclusion Bodies ${ }^{[1]}$}

Inclusion bodies are classified as follows.

1. Physiological inclusion bodies

2. Inclusion bodies in infections

A. Inclusion bodies in viral condition

i. Intra cytoplasmic inclusions

ii. Intra nuclear inclusions

B. Inclusion Bodies seen in bacterial infections.

C. Inclusion Bodies seen in fungal infections.

3. Inclusion bodies in neoplasms.

4. Inclusion bodies in autoimmune diseases.

5. Inclusion bodies seen in blood dyscrasias.

6. Inclusion bodies seen in cystic lesions.

7. Physiological inclusion bodies

Odland bodies ${ }^{[2]}$

Also called as keratinosomes or lamellar bodies. Odland bodies are small in size and granular. They are membrane-bound vacuoles found in the cytoplasm of skin keratinocytes. These are seen in the upper stratum spinosum and stratum granular cell layers which are rich in glycolipids.

\section{Weibel Palade bodies}

They are small secretory vesicles present in endothelial cells that contains von Willebrand factor ${ }^{[3]}$. They are cigar-shaped, surrounded by a standard lipid bilayer membrane. In some endothelial cells, especially in the lungs, factor VIII is stored in Weibel-Palade bodies along with von Willebrand factor ${ }^{[4]}$.
Infection inclusion bodies $\rightarrow$ Inclusion bodies in viral condition $\rightarrow$ Intra cytoplasmic inclusions

\section{Councilman Bodies}

Otherwise called as Councilman hyaline body or apoptotic body. It is seen most commonly in Liver biopsy patients with viral hepatitis, Yellow fever and other viral hemorrhagic fevers ${ }^{[5]}$. It is an eosinophilic globule that represents a hepatocyte. It is similar to mallory bodies but twisted rope appearance will not be present.

\section{Henderson Peterson bodies}

Otherwise called as Molluscum Contagiosum. It is caused by Pox virus ${ }^{[6]}$. They have a pearly appearance. The lesion is found in face, neck, arms, legs, abdomen, and genital area. It is rare in hands and foot. It is a contagious disease with benign raised bumps or lesions on the upper layers of your skin.

Intra nuclear inclusions

Cowdry Type- A

Cowdry Type A are round eosinophilic material surrounded by a clear halo. They are acidophilic material of droplet-like. Commonly seen in gingivostomatitis, conjunctivitis and Chicken Pox. They contain intact and disrupted virions and push darkly stained host cell chromatin to the edges of the nucleus ${ }^{[7]}$.

\section{Lipschutz bodies}

Lipschutz bodies are eosinophilic nuclear inclusions having enlarged nuclei and surrounded by a clear halo. They have enlarged nuclei. Commonly seen in varicella and herpes simplex ${ }^{[8]}$. 


\section{Cowdry Type- B}

Cowdry Type B are inclusion bodies with intranuclear eosinophilic amorphous bodies. They are surrounded by a clear halo without other nuclear changes during early stages of development of the inclusion. They are seen in neural cells.

Owl's eye ${ }^{[9]}$

Owl's eye can be used to describe the nuclei of cells infected by CMV and it can also be used to describe the nuclei of Reed-Sternberg cells

Inclusion Bodies seen in bacterial infections.

Dohle bodies $^{[10,11]}$

Dohle bodies are inclusion bodies which are small, round or oval in size and pale blue to grey in structures. They are usually found at the periphery of the neutrophil. Dohle bodies are seen in typhoid, diphtheria and tuberculosis.

Inclusion Bodies seen in fungal infections.

Asteroid bodies ${ }^{[12,13]}$

Asteroid bodies are inclusion bodies which are stellate, periodic acid-Schiff positive eosinophilic material that surround the organisms.

\section{Toto bodies}

These are inclusion bodies which are of eosinophilic structures which resemble the cells of the superficial cell layer of the oral epithelium. This inclusion body is seen in conditions like epulis fissuratum and inflammatory hyperplastic gingivitis.

\section{Inclusion bodies in neoplasm.}

Wagner- Meissner body ${ }^{[14]}$

Also called as Meissner corpuscles. Wagner Meissner corpuscles are specialized mechanoreceptors located in the dermal papillae. Wagner Meissner corpuscle like structures are occasionally a component of certain types of tumours, such as melanocytic nevus and neurofibroma. Seen in von Recklinghausen's disease of skin, neurofibroma.

\section{Verocay bodies}

These inclusion bodies are histological feature of schwannomas. They have a pattern of Antoni type $A$ in which tumour cells form alternating parallel rows of nuclear palisades separated by regions of acellularity. They are seen in benign nerve sheath tumour, Schwannoma and other diseases.

\section{Psammoma bodies}

They are inclusion bodies which are concentric lamellated calcified structures. It is seen in diseases of papillary thyroid carcinoma, meningioma, and papillary serous cystadenocarcinoma of ovary but have rarely been reported in other neoplasms and nonneoplastic lesions. Seen in numerous benign and malignant epithelial and connective tissue tumours such as psammomatoid meningioma, psammomatoid juvenile ossifying fibroma, psammomatoid melanotic schwannoma, cystadenocarcinoma.

\section{Russell bodies}

Russell bodies are globular or spherical cytoplasmic inclusions which is eosinophilic that accumulate in the rough endoplasmic reticulum of mature plasma cells. These plasma cells containing Russell bodies are also known as Mott cells. Seen in chronic inflammatory granulomata, multiple myeloma, plasmacytoma, helicobacter pylori infection, periapical granuloma.

\section{Pustulo- Ovoid bodies ${ }^{[15]}$}

These inclusion bodies are larger granules surrounded by a clear halo. It has coalescing granules. It is seen in granular cell tumours.

\section{Kamino bodies}

Kamino bodies are eosinophilic globoids. Which are observed microscopically. It is a benign melanocytic nevus, a type of skin lesion, affecting the epidermis and dermis. Kamino bodies are rare in melanoma. They are hyaline structures that stain positively with periodic acid-Schiff and trichrome. Kamino bodies are commonly found in the dermo-epidermal junction

\section{Dutcher bodies}

Dutcher bodies are PAS-positive, smooth, membranebound and surrounded by clumped chromatin, immunoglobulin protein, diastase-resistant nuclear pseudoinclusions found in plasma cells. It was described by Dutcher and Fahey in Waldenstrom macroglobulinemia. They have been observed in cases of myeloma. It is seen in chronic synovitis and large Bcell lymphoma and multiple myeloma. It stains with Wright- Giemsa stains and periodic acid Schiff's stains.

\section{Inclusion Bodies in autoimmune diseases.}

\section{Civatte bodies}

They are seen as rounded, homogenous, eosinophilic masses on routine haemotoxylin and eosin staining lying in the deeper parts of epidermis/epithelium and more frequently in dermis/connective tissue. They are also known as colloid bodies, or hyaline bodies. It is derived from basal cells and connective tissue elements from the basement membrane zone 


\section{Haematoxylin bodies}

This inclusion body is homogeneous and dense with necrotic loci and it contains dense chromatin, basophilic particle, easily stainable with haematoxylin. It is seen in SLE, Hodgkin's disease, rheumatic disease etc.

\section{Schaumann bodies}

Schaumann inclusion bodies are concentrically lamellated structure which is large and is seen in the cytoplasm of the giant cells, presence of calcium and phosphorus and small quantities of iron in Schaumann bodies. It is seen in Sarcoidosis, tubercular granuloma, hypersensitive pneumonitis.

\section{Inclusion bodies seen in blood dyscrasias.}

\section{Heinz bodies}

Heinz bodies are denatured globin which is irregular, small and present as deep purple granules and represent the end-product of oxidative degradation of haemoglobin. Heinz bodies may be detected postsplenectomy, with oxidative haemolysis and in patients with unstable

\section{Howell- Jolly bodies}

Howell-Jolly bodies are round, smooth, almost pyknotic, dark-purple bodies which has ring like appearance that mimics parasites. It is seen in Pernicious anemia and leukaemia with megaloblastic anemia. Howell Jolly bodies will be represented as DNA remnants.

\section{Inclusion bodies seen in cystic lesions}

\section{Rushton bodies/ Hyaline bodies}

Rushton bodies are seen commonly in radicular cyst, plexiform ameloblastoma. It is stained in Mallory aldehyde fuchsin, periodic acid-Schiff, Gomori stains, Papanicolaou and Orcein. Rushton bodies represent as a curved or straight glassy structure.

\section{Conclusion:}

The inclusion bodies give a clue in diagnosis of oral lesions. Few of them are pathognomonic for specific lesions and can be readily identified through microscope.

\section{References:}

1. Meena Kulkarni, Tripti Agrawal, Histopathologic bodies: An insight, Varsha Dhas Department of Oral pathology and Microbiology, Dr. D. Y. Patil Dental College and Hospital, DPU Pimpri, Pune, India
2. Rajiv Joshi, Indian Dermatol Online; Learning from eponyms: George F. Odland and Odland bodies J. 2014 Jul-Sep

3. Lowenstein $\mathrm{CJ}$, Morrell $\mathrm{CN}$, Yamakuchi $\mathrm{M}$. Regulation of weibel palade body exocytosis. Trends Cardiovasc 2005;15:302-8.

4. Daniel J. Metcalf, Thomas D. Nightingale, Helen L. Zenner, Winnie W. Lui-Roberts, Daniel F. Cutler, Formation and function of Weibel-Palade bodies Journal of Cell Science 2008 121: 19-27; doi: $10.1242 /$ jcs.03494

5. Am J Pathol. 1966 May; 48(5): 755-767. MCID: PMC1907261PMID: 4287129 The ultrastructure of acidophilic "Councilman-like" bodies in the liver. F. M. Klion and F. Schaffner

6. Sangster A. Non-glandular theory of origin of Molluscum contegiousum. Lecture on skin diseases at Charing cross Hospital;1880.

7. Eyons AS, Kaslow RA. Viral Infections of Humans: Epidemiology and Control. British Journal of Biomedical Science. June 1, 1998;1029;813.

8. Antonio C, Slootweg PJ. Pathology of the head and neck; Vesiculobullous Diseases,1999; 72.

9. Matt es FM, McLaughlin JE, Emery VC, Clark DA, Griffi ths PD.Histopathological detection of owl's eye inclusions is still specific for cytomegalovirus in the era of human herpesviruses 6 and 7.Clin Pathol 2000;53:612-4.

10. Weiner W, Topley E. Dohle bodies in the leucocytes of patients with burns. J Clin Pathol 1955;8:324-8.

11. Easton JA, Fessas $C$. The incidence of dohle bodies in various diseases and their association with thrombocytopenia. Br J Haematol 1966;12:54-60

12. Rajendran $R$, Sivapathasundharam.B. Shafer's Textbook of Oral Pathology. 6th ed. Mycotic Infections of Oral Cavity. p. 369.

13. Berson SD, Brandt FA. Primary pulmonary sporotrichosis with unusual fungal morphology. Thorax 1977;32:505-8

14. Ben ZP. Head and neck surgical pathology; Soft Tissue Pathology of Head and Neck. 1997; 397-419.

15. Ohno J, Iwahashi T, Ozasa R, Okamura K,Taniguchi $K$. Solitary neurofi broma of the gingival with prominent differentiation of Meissener bodies: $A$ case report. Diagn Pathol 2010; 5: 61.

16. Simansky DA, Aviel-Ronen S, Reder I, Paley M, Refaely Y, Yellin A. Psammomatous melanotic schwannoma: Presentation of a rare lung tumor. Ann Thorac Surg 2000; 70: 